\title{
Incidente de Múltiples Víctimas, Una Prueba Para un Sistema de Emergencias Nuevo, Sur de Ecuador, 2014
}

\author{
${ }^{1}$ Leonardo Serrano, ${ }^{2}$ Daniel Sacoto, ${ }^{3}$ Hernán Sacoto, ${ }^{4} J u a n$ Carlos Salamea
}

\section{RESUMEN}

Antecedentes: A medida que las ciudades crecen, la gestión de seguridad es más compleja, El Gobierno ha invertido más de 6 veces en seguridad, la creación del sistema integrado de seguridad SIS ECU-911, articula a todos los organismos de respuesta ante cualquier situación de contingencia. ${ }^{1}$ Pese a ello, las estadísticas de seguridad vial de la agencia nacional de tránsito (ANT) indican un incremento de accidentes de tránsito. ${ }^{2}$ El presente estudio intenta mostrar las fallas que aún tiene el sistema de seguridad en cuanto a la coordinación operativa de respuesta.

Diseño: Observacional descriptivo: reporte de caso.

Resultados: El incidente produjo 44 lesionados. EI SIS ECU-911 fue alertado. La primera ambulancia arribó a 45 minutos. Se realizó triage y manejo inicial por paramédicos, sin embargo esta no pudo tomar control sobre las demás víctimas, produciendo una evacuación no sistemática del resto de lesionados mediante el usos de vehículos particulares. El SIS ECU-911 falló, provocando retrasos en la transmisión de la información entre ellos y el centro de trauma. En los hospitales de referencia, se produjo la llegada imprevista de los lesionados sin una preparación previa del personal; Si bien se pudo hacer un correcto triage inicial intrahospitalario, se pudo evidenciar la falta de recursos materiales. En el presente caso, el enfermo es el automotor y su chofer, con una lista de problemas: 1. Falta de mantenimiento. 2. Imprudencia e impericia. 3. Exceso de pasajeros.

Conclusiones: EI SIS ECU-911 en nuestro país es nuevo. Existen deficiencias, las que deben resolverse; sin embargo consideramos que se dirige por buen camino. La implementación de simulacros periódicos ayudaría a detectar falencias del sistema con el fin de tener una sociedad más

\footnotetext{
${ }^{1,2}$ Estudiante de Medicina, ${ }^{3}$ Profesor de Cirugía, ${ }^{4}$ Surgeon

${ }^{1}$ Department of Liga Académica de Trauma y Emergencias Universidad de Cuenca, Ecuador

${ }^{2}$ Department of Liga Académica de Trauma y Emergencias Universidad del Azuay, Ecuador

${ }^{3}$ Department of Cirugía, Universidad del Azuay, Ecuador

${ }^{4}$ Department of Cirugía Trauma y Emergencias, Hospital Vicente Corral Moscoso, Ecuador
}

Corresponding Author: Juan Carlos Salamea, Surgeon Department of Cirugía Trauma y Emergencias, Hospital Vicente Corral Moscoso, Ecuador, Phone: 59374096597 e-mail: jsalamea@gmail.com segura. Igualmente campañas de prevención, como las que actualmente se desarrollan con el proyecto de las ligas de trauma pudieran evitar accidentes futuros.

Palabras claves: Accidente de tránsito, Centro de trauma, Servicios médicos de urgencia, Planes de emergencia, Sistema médico de emergencia.

How to cite this article: Serrano L, Sacoto D, Sacoto $H$, Salamea JC. Incidente de Múltiples Víctimas, Una Prueba Para un Sistema de Emergencias Nuevo, Sur de Ecuador, 2014. Panam J Trauma Crit Care Emerg Surg 2015;4(1):30-35.

Source of support: Nil

Conflict of interest: None

\section{ABSTRACT}

Background: As cities grow, managing security is more complex, the government has invested more than 6 times in security, the creation of the integrated security system SIS ECU-911, articulates all agencies to respond to any situation contingency. ${ }^{1}$ Nevertheless, road safety statistics of national transit agency (ANT) indicate an increase in traffic accidents. ${ }^{2}$ The present study attempts to show the flaws that still has the security system in terms of operational coordination of response.

Design: Observational descriptive: a case report.

Results: The incident occurred 44 injured. The SIS ECU-911 was alerted. The first ambulance arrived 45 minutes. Triage and initial management was performed by paramedics, but this could not take control of the other victims, producing a nonsystematic evacuation of the remaining injured through the use of private vehicles. The SIS ECU-911 failed, causing delays in the transmission of information between them and the trauma center. In the reference hospitals, there was the unexpected arrival of the injured without prior training of personnel; while it could make proper initial in-hospital triage, was evident lack of material resources. In this case, the patient is the motor and driver, with a list of problems: (1) Lack of maintenance, (2) imprudence and inexperience and (3) excessive passengers.

Conclusion: The SIS ECU-911 in our country is new. There are shortcomings, to be resolved; however, we consider that targets on track. The implementation of periodic drills helps to detect flaws in the system in order to have a safer society. Equally, prevention campaigns, such as those currently being developed with the draft leagues trauma, could prevent future accidents.

Keywords: Accidents, Traffic, Trauma center, Emergency plans, Emergency medical services.

Paper presented in research competition in the PTS Congress, Panama, 2014. 


\section{INTRODUCCIÓN}

Los Incidentes de Múltiples Víctimas son eventos complejos, que ponen a prueba a los sistemas de emergencias médicas, y son especialmente complicados para un sistema relativamente nuevo como el SIS ECU 911 en el Ecuador. Representan un reto que involucra a todos los profesionales de la salud, personal administrativo, autoridades, estudiantes, Universidades de Ciencias de la Salud y población en general debido a que debieran estar capacitados, preparados y organizados de tal manera que se pueda dar el mayor beneficio al máximo número de víctimas.

Las colisiones de tránsito, mal llamados accidentes de tránsito, ${ }^{3}$ son una de las principales causas de morbimortalidad en el país. Según la Agencia Nacional de Tránsito hasta Noviembre del 2013 se registraron 24759 accidentes de transito, siendo la impericia del conductor y las fallas mecánicas del automtor, las principales causas relacionadas. ${ }^{4}$

En el Reporte del Estado Global sobre la seguridad de las vías de la Organización Mundial de la Salud (OMS) publicado en el 2013, el Ecuador ocupa el segundo lugar en mortalidad por colisiones de tránsito con una tasa de 28 muertes por cada 100000 habitantes. ${ }^{5}$

El servicio integrado de seguridad (SIS ECU 911) creado hace 2 años es un modelo de gestión que integra a todas las instituciones de atención de emergencias, como Policía, Fuezas Armadas, Bomberos, Salud, Cruz Roja, no solo par optimizar recursos si no para abarcar la mayor atención ciudadana, con el objetivo de brindar atención de emergencias a través de un número único. La ciudad de Cuenca cuenta con el hospital público de referencia (Hospital Vicente Corral Moscoso) en donde se encuentra el centro de trauma y emergencias. De igual manera entre los hospitales de referencia se encuentra el Hospital del Seguro Social (Jose Carrasco Arteaga).

Se describe un caso de acciente con multiples víctimas producto de un volcamniento de autobus, en el que se vieron involucradas 44 pasajeros. Se analizan las debilidades y fortalezas en la organización y coordinación del nuevo Sistema de Emergencias que funciona en el Sur del Ecuador, en el caso de un incidente de múltiples víctimas. Se plantean oportunidades para mejorar el sistema y evitar morbimortalidad prevenible. Se describe con detalle el proceso realizado tanto a niverl prehospitalario como en el Hospital publico Vicente Corral Moscoso (HVCM).

\section{MÉTODOS}

Entre el 5 y 10 de junio un equipo de estudiantes de la ligas de trauma, ${ }^{1,2}$ reviso formularios, historias clínicas, reportes policiales respecto del accidente del vehículo motorizado tipo bus que se sucinto en el sector de Shiña, provincia del Azuay. El día del incidente los dos primeros investigadores estuvieron presentes en la guardia del HVCM. Pudieron constatar las acciones ejecutadas por la nueva red integral de seguridad (SIS ECU 911) en los escenarios pre-hospitalario, trasporte y hospitalario. Eso involucra las acciones y decisiones que se tomaron dentro y fuera del centro de trauma.

Los días posteriores al incidente se acudió al centro de trauma (HVCM), al instituto del seguro social (IESS) y al centro de gestión de riesgo para la recolección de información relevante en un formulario preestablecido para el efecto. Posteriormente se realizaron el análisis de las historias clínicas, y reportes policiales. Los datos se presentan en tablas de frecuencias.

\section{RESULTADOS}

Domingo 09-02-2014, 17:52 el SIS ECU 911 Zona 6 recibe una llamada de alerta de un volcamiento de un autobús publico con 44 pasajeros desde una zona, ubicada a una hora y media de la Cuidad de Cuenca. Se activa la red de emergencias y se envían los recursos que en ese momento se consideraron necesarios (tres ambulancias) (Tabla 1). La primera ambulancia llega a la escena aproximadamente a los 45 minutos de después del incidente; Una vez en a escena, se asegura el lugar, y los socorristas (Voluntarios) realizan el triage y traslado de lesionados. Al sentirse sobrepasados, solicitan mas ambulancias y apoyo, sin embargo hasta su llegada, se improvisan traslados en vehículos particulares. El sistema de comunicación entre socorristas el SIS ECU 911 y Hospitales estubo disfuncional, por lo que se utilizaron redes sociales, lo que provocó un retraso considerable en la preparación de los equipos de trauma en los servicios. ${ }^{6}$

Tabla 1: Personal y recursos despachados el día del evento

\begin{tabular}{ll}
\hline Institución & Recursos \\
\hline Cruz Roja Ecuatoriana, Junta Provincial & 2 Ambulancias \\
del Azuay & 6 Paramédicos \\
Policía Nacional del Ecuador & 1 Patrullero \\
& 3 Policías \\
Comisión de Tránsito del Ecuador & 1 Patrullero \\
& 3 Agentes \\
Ministerio de Salud Pública & 3 Ambulancias \\
Benemérito Cuerpo de Bomberos & 9 Paramédicos \\
Voluntarios de Cuenca & 1 Ambulancia \\
& 1 Vehículo de rescate \\
Fuerzas Armadas Ecuatorianas* & 6 Bomberos \\
& 1 Camioneta \\
\hline
\end{tabular}

*Los militares se encontraban en el sitio por coincidencia;

Fuente: Secretaria de Gestión de Riesgos, República del Ecuador Sala Situacional de la Provincia del Azuay; elaboración: Autores 
Tabla 2: Lesiones de 20 pacientes entregados al Hospital Vicente Corral Moscoso

\begin{tabular}{ll}
\hline Lesiones & Total \\
\hline Contusión simple partes blandas & 9 \\
Heridas partes blandas & 9 \\
TEC & 8 \\
Trauma tórax & 1 \\
Trauma miembros & 9 \\
Fracturas & 4 \\
\hline
\end{tabular}

Fuente: Base de datos Hospital Vicente Corral Moscoso; Elaboración: Autores

A las 19:20, se entregaron los primeros pacientes en los hospitales de la ciudad, Hospital Vicente Corral Moscoso: 20 víctimas (Tabla 2); y al Hospital José Carrasco Arteaga 24 pacientes. Un paciente falleció en la escena y 43 sufieron lesiones de variada gravedad. El tipo de lesión de cada paciente se detalla en la tabla. ${ }^{2}$ La información es completada en el HVCM, en donde de los 20 pacientes atendidos, 14 fueron dados de alta el mismo día, 6 pacientes ingresaron a observación, y de ellos 4 necesitaron cirugía para el control de sus lesiones. De estos 1 requirió manejo en cuidados intensivos, el mismo que finalmente falleció a los 12 días debido a falla multi-orgánica. Este evento se presenta como un historial clínico en donde el enfermo es el automotor. El paciente es un autobus marca Hino Modelo GD1JLTZ, perteneciente a la Cooperativa de transportes urbanos 'Zhiña', 14 años de edad, nacido en Japon y actualmente reside en Cuenca. Entre sus antecedentes, figuran la ultima revisión vehicular hace 5 años y matricula caducada. Falta de mantenimiento mecánico regular. El conductor registra desde el 2001, 6 sanciones, con multas por contravenciones. Se desconoce información respecto del conductor como edad, tipo de licencia, consumo de estupefacientes, alcohol, sedantes, horas de sueño anteriores al evento, pues el mismo se dio a la fuga. 'Según uno de los pasajeros el conductor transitaba a gran velocidad y la unidad perdió los frenos' ${ }^{6}$ El examen físico del automotor reveló todas las llantas lisas.

\section{DISCUSIÓN}

Según el informe de la Organización Mundial de la Salud (OMS), Ecuador es el segundo país en Sudamérica con el mayor índice de muertes por accidentes de tránsito. Esta cifra la corroboran estadísticas del Instituto Nacional de Estadística y Censos (INEC) que identifican estos siniestros como la primera causa de fallecimiento en los hombres y como la quinta a nivel general. La Agencia Nacional de Tránsito (ANT) registra que cada 20 minutos ocurre un accidente de tránsito en el país y cada cuatro horas alguien muere por esta causa. ${ }^{7} \mathrm{El}$ director ejecutivo de la Comisión Interinstitucional de
Educación, Seguridad y Prevención vial (Covial) explica que en Ecuador cada 18 días hay accidentes denominados como siniestros de magnitud, es decir, aquellos en que hay más de 15 víctimas, ya sean heridos o muertos. ${ }^{1}$

Si bien la iniciativa de contar con un sistema integrado de seguridad nuevo es loable, el presente caso ilustra varias debiliades del sistema:

- Sub valoración del incidente en el SIS ECU 911 con la consecuente toma de decisiones inapropiadas.

- Proceso de triage en escena heterogéneo y rudimentario.

- Sistema de comunicación deficiente.

- Protocolo de alerta hospitalaria ineficiente.

- Cotización en los servicio de emergencia.

Teniendo esto en mente, y considerando el aumento del parque automotor, el crecimiento poblacional, y el mejoramiento de las vias a lo largo y ancho de la nación, es muy probable que se incrementen los incidentes en los cuales se involucren más de un paciente, sobre todo en servicios de transporte masivos. A estos incidentes que involucran más de una víctima pero que no sobrepasan los recursos y capacidad de los Sistemas de Emergencia se los llama Incidentes con Múltiples Víctimas, para diferenciarlos de los que sobrepasan la capacidad de los mismos, a los que se los conoce como Incidente Masivo o Desastre. ${ }^{3,8}$

En estos casos las prioridades y el manejo cambian con respecto a otras situaciones de emergencia indicidual, con el fin de salvar a la mayor cantidad de lesionados, y no centrarse en los más graves debido a la limitación de recursos. $^{3,8}$

El manejo del incidente con múltiples víctimas, debe ser integral y puede ser resumido en las siguientes fases:

- Preparación y prevención

- Fase prehospitalaria

- Transporte

- Fase hospitalaria $3,8,9$

Un incidente de múltiples víctimas o un desastre pueden suceder en cualquier momento, aunque no sean comunes. Su manejo debe ser planificado, para ello les importante estar preparado. Esto se logra con la planificación de simulacros periódicos; ${ }^{10,11}$ En nuestra ciudad los simulacros no se realizan periódicamente, el último fue realizado en el 2012. Esto bien puede explicar el desorden e imrpovisación en estas situaciones. Los simulacros sirven para identificar debilidades del sistema, los cuales son dinámicos en el tiempo, y nos permiten orientar políticas para su corrección. En nuestro estudio se identificaron claramente debilidades, especialmente en lo que respecta a las comunicaciones entre los involucrados para el cuidado de lesionados. 
Para estas situaciones debe existir personal entrenado en métodos de triage como el START y JumpSTART o el método SALT con el fin de atender y transportar en el prehospitalario de manera eficiente con los limitados recursos que existen, en este incidente al parecer se realizó un triage pero no se conoce con exactitud cuál fue el método utilizado. ${ }^{3,12,13}$ Para el traslado de los pacientes se debió elegir el lugar apropiado y más cercano dependiendo de las lesiones, consideramos que en este punto existieron grandes deficiencias debido a que a el Centro de Trauma del HVCM llegaron varios pacientes con lesiones menores que debieron ser llevados a centros de menor complejidad (Tabla 2); otro error fue el solicitar que vehículos particulares sean quienes trasladen a los pacientes sin antes ser evaluados por un profesional de la salud quien decida a donde deberían ser trasportados y solo debieron trasladar a pacientes menos severos, reservando las ambulancias para los graves. ${ }^{12,13} \mathrm{En}$ relación con la fase hospitalaria, cada hospital debe contar con su plan en caso de incidentes múltiples, el cual todo el personal debe conocerlo; la preparación previa se realizó correctamente en el HVCM pese a que la información que recibía no era completa y oportuna se despejó la sala de emergencias, preparó quirófanos y sala de trauma, además se llamó y alistó a especialistas necesarios. Sin embargo existieron algunas fallas que consideramos deben mejorarse, existen falta de espacios adecuados para tratar este tipo de incidentes el hospital debe estar diseñado de tal manera que existan salas para triage, pacientes con lesiones menores, víctimas más graves, salas de espera para familiares, sala de prensa, sin embargo debido a la falta de estas, en la sala de emergencias hubo desorganización entorpeciendo la labor de los médicos, incluso lograron ingresar familiares y prensa saturando el lugar, para evitar esto se debería capacitar al personal de seguridad del hospital. ${ }^{9}$ Otro punto es la falta de suministros y organización de los mismos, una buena opción es tener preparado material especial para estas situaciones como camillas plegables o colchones además los suministros deben estar organizados de tal manera que existan estuches con equipos para manejo de vía aérea, circulación, etc, lo cual no existe en el HVCM; otro falla es la falta de preocupación en relación con el material de bioprotección, en esta ocasión ningún miembro del equipo utilizó todos los materiales básicos de bioprotección. ${ }^{8}$

A la llegada de los pacientes a la sala de emergencias del HVCM se realizó correctamente un triage inicial, ${ }^{8,9}$ sin embargo al llegar varios carros particulares y conjuntamente el otro incidente de tránsito muchos de los pacientes ingresaron sin antes ser evaluados por lo que la atención se entorpeció.
La falta de una persona encargada solo para dar información a los familiares y medios de comunicación provocó que muchos familiares tengan crisis emocionales, sobre todo en la sala de pediatría además de desinformación. ${ }^{9}$

Por último se debe tener un buen sistema de registro y realizar una evaluación de lo sucedido, considerándola como una oportunidad para mejorar y no con un objetivo punitivo.

También se debe considerar la necesidad de tratamiento psicológico para sobrevivientes, familiares y personal que presente síntomas de trastorno por estrés agudo o posteriormente estrés postraumático.

\section{CONCLUSIONES}

Si bien el Ecuador es un pais considerado pacifico, en donde la violencia no alcanza niveles alarmantes como en paises vecinos, es preocupante que pese al incemento de penas por infracciones de tránsito, sigan transitando en nuestras carreteras verdaderas bombas de tiempo. Claramente el problema no se solucionaría solo con multas y carcel, médidas con resultados a corto o mediano plazo, sino con un proceso de culturización desde los primeros años escolares, que generen cambios de conducta voluntarios, y permanentes. Obiamente, este es un proceso con resultados a largo plazo. El sistema de Emergencias en nuestro país, de la manera como está actualmente estructurado, es relativamente nuevo, y todavía tiene defectos que deben y pueden ser resueltos, sin embargo consideramos que se dirige por buen camino. La implementación de simulacros periódicos ayudaría a organizar y planificar mejor en caso de que se produzca algún accidente de múltiples víctimas o en un desastre.

\section{REFERENCIAS}

1. Ministerio Coordinador de Seguridad [Internet]. ECU 911 ante lasemergenciasen salud, Quito;2012.p. 18. [Consultado2014 Abr 24]. Disponibleen: Availableat: http://www.seguridad.gob.ec/ wp-content/uploads/downloads/2012/08/revista3Final.pdf.

2. Agencia Nacional De Transito DQ. Siniestros por causas probables a nivel nacional a agosto-2014 Comparativo Mensual Agosto Número de Siniestros por Causas Probables. Quito; 2014 p. 14. Available at: http://www.ant.gob.ec/index. php/noticias/estadisticas\#.VCyVHIF5MrU.

3. Colegio Americano de Cirujanos, Comité de Trauma. Soporte vital básico y avanzado en el trauma prehospitalario. Séptima ed. Barcelona: Elsevier España; 2012.p.23.

4. Agencia Nacional de Tránsito. Estadísticas de transporte terrestre y seguridad vial; 2014.

5. Organización Mundial de la Salud. Informe sobre la situación mundial de la seguridad vial 2013. Apoyo al decenio de acción; 2013.

6. Centro Zonal Integrado de Seguridad ECU-911 Austro. Twitter ECU-911 Austro; 2014.

7. Agencia Nacional De Transito DQ. Dirección de Estudios y Proyectos Comparativo Mensual Agosto 2013-2014 Número 
de Fallecidos en Sitio por Provincia [Internet]. Quito; 2014. p. 14. Available at: http://www.ant.gob.ec/index.php/ descargable/file/2463-fallecidos-agosto-2014.

8. Colegio Americano de Cirujanos, Comité de Trauma. Soporte vital avanzado en trauma para médicos. Novena edición; 2012. p. 6.

9. Tintinally J, Stapczynski J, Jhon O, Cline D, Cydulka R, Meckler G. Tintinalli's Emergency Medicine: a comprehensive study guide. 7th ed. New York: McGraw Hill Professional; 2010. p. 27-60.

10. Hsu EB, Jenckes MW, Catlett CL, Robinson KA, Feuerstein C, Cosgrove SE, et al. Effectiveness of hospital staff mass- casualty incident training methods: a systematic literature review. Prehosp Disaster Med. Cambridge University Press; 2012 Jun;19(03):191-199.

11. World Health Organization. Mass casualty management systems strategies and guidelines for building health sector capacity. Geneva: WHO Document Production Services; 2007. p. $16-20$.

12. Greaves I, Porter K. Oxford handbook of prehospital care. Oxford: OUP; 2006. p. 575-618.

13. Advance life support group. Major incident medical management and support: the practical approach at the scene. Londres: BMJ books; 2011. p. 91-102. 


\section{INVITED COMMENTARY}

\section{A Critical Appraisal of an Incident with Multiple Victims in a New Emergency System}

In first place, I would like to thank you for the opportunity on commenting this very interesting manuscript from Cuenca, Ecuador. I believe the decision of having this paper published in the Panamerican Journal of Trauma Critical Care \& Emergency Surgery (PAJTCCES) was made very right, as this is an important on going picture of the current emergency system in Ecuador.

Here, authors bring to light a critical appraisal of an incident with multiple victims happened in the south zone of the country. Although a couple of important details are still missing due to an incipient system, a local illustration about country's emergency system is drawn. This kind of manuscript is an important tool for government and institutions enrolled in emergency care to be used as a way for achieving the state-of-the-art emergency system and should be encouraged to be performed in each country with no mature emergency system. With simple reports like this, essential cobblestones in the emergency system can be reinforced, mistakes and failures revealed and suggestions for improvements can be done with the final objective of upgrading the whole emergency system and service.

In this specific manuscript entitled 'Incidente de múltiples víctimas, una prueba para un sistema de emergencias nuevo, sur de Ecuador, 2014', this group of authors picked up an incident with 44 victims of a bus rollover crash and detailed all the rescue and hospital treatment since the first emergency team activation call until post crash information. The rationale for writing the aforementioned paper is pretty much plausible since the emergency system in Ecuador is new. Local road crash statistics are increasing each year, being responsible for one of the main causes of death in the country. This country is second in the world in road crash fatalities according to the 2013 OMS report.

Here in the authors recognize the importance of having an integrated emergency system but also recognize several week points of the nascent system. Trauma league medical students investigated manifold aspects before, during and after the incident and found that although the emergency system was activated, there was an important delay on sending the rescue team as well their arrival at the crash scene- 45 minutes after the incident. The triage method was also pointed out as a week point of the system. In this case, it is important to remember that for complex incidents such this one, the emergency system is not only the activation of the rescue team and transportation for a referral center but the enforcement of prevention in trauma, disaster and mass casualty response preparedness. Establishing and implementing a disaster plan before the incident occurs is also the core for a well succeeded disaster response in such incidents. With an established and well-known disaster plan by all involved parts, common mistakes in triage such as super-triage and under-triage tend to happen less frequently. The paramount lesson here is to have a flowchart specifying functions and activities before the disaster or incident occurs. After this, training, training and training, based on the new created incident command system is mandatory.

From a reading of the manuscript, it looks like the emergency system in Ecuador is getting better but is still evolving. The need for emergency and disaster plans implementation and coordination is beginning.

Another interesting focus was given to the cause of the incident. The authors performed an investigation regarding the mechanical condition of the motor vehicle (bus) involved in the crash, demonstrating that not just the emergency system but all the infrastructure around it with prevention aspects play an important role and need improvements.

This article shows as strong points the usage of trauma leagues as a force for promoting trauma education, prevention and research, identification of mistakes in a newborn emergency system and brings suggestions for local improvements. However, this paper is a local picture of a system that plays a role in all cities of the country and may not be the same for a nationwide territory. Also, excluding the big value that this article brings on, if research and publishing is a field that authors want to improve, modification in manuscript structure and design must be achieved. Personal comments, for instance, must be left for the discussion section not in the results section.

As a final point, I would like to congratulate the authors on a very good job and thank them for submitting it to PAJTCCES.

Bruno M Pereira

Associate Professor, Department of Surgery University of Campinas, Brazil

Co-Chair, SPT Membership Committee 